\title{
Real-time Visual Analysis of Microvascular Blood Flow for Critical Care
}

\author{
Chao Liu * \\ Hernando Gomez ${ }^{\dagger}$ \\ Srinivasa Narasimhan * \\ Artur Dubrawski * \\ Michael R. Pinsky ${ }^{\dagger}$ \\ Brian Zuckerbraun ${ }^{\dagger}$
}

\begin{abstract}
Microcirculatory monitoring plays an important role in diagnosis and treatment of critical care patients. Sidestream Dark Field (SDF) imaging devices have been used to visualize and support interpretation of the microvascular blood flow. However, due to subsurface scattering within the tissue that embeds the capillaries, transparency of plasma, imaging noise and lack of features, it is diffcult to obtain reliable physiological data from SDF videos. Therefore, thus far microcirculatory videos have been analyzed manually with significant input from expert clinicians. In this paper, we present a framework that automates the analysis process. It includes stages of video stabilization, enhancement, and micro-vessel extraction, in order to automatically estimate statistics of the micro blood flows from $S D F$ videos. Our method has been validated in critical care experiments conducted carefully to record the microcirculatory blood flow in test animal subjects before, during and after induced bleeding episodes, as well as to study the effect of fluid resuscitation. Our method is able to extract microcirculatory measurements that are consistent with clinical intuition and it has a potential to become a useful tool in critical care medicine.
\end{abstract}

\section{Introduction}

Microcirculation takes place in part of the circulatory system embedded in tissue that involves the smallest vessels and where diffusion of nutrients and oxygen into the cells and removal of $\mathrm{CO}_{2}$ and waste from the cells take place. Monitoring of microcirculation is useful for diagnosing of vascular conditions and in monitoring patients for cardiorespiratory insufficiency.

Sidestream Dark Field (SDF) [6] video imaging was developed as a non-invasive imaging approach for real-time visualization of superficial microvascular flow. However, analysis of these videos is currently limited by manual

\footnotetext{
*Carnegie Mellon University, The Robotics Institute. Email:\{chao.liu, srinivas, awd\}@cs.cmu.edu

$\dagger$ University of Pittsburgh, School of Medicine. Email:\{gomezh, pinskymr, zuckerbraunbs\}@upmc.edu
}

or semi-manual operation and coarse sampling techniques, which makes quantitative analysis of microcirculatory status and response to disease and treatment difficult and subjective [7]. We aim to remedy that. One of the portable SDF imaging devices is shown in Fig. 1(a). As depicted in Fig. 1(c), illumination is provided by the green lightemitting diodes (LEDs) arranged in a ring formation. The wavelength $(\lambda=530 \mathrm{~nm})$ of the illumination is chosen to maximize light absorption by the red blood cells (RBCs). The tissue embedding the capillaries scatters and reflects the illumination back to the camera, making the capillaries imaged as dark curvilinear structures against the brighter background. The LEDs and the lens system are optically isolated to prevent the illumination generated by the LEDs from contaminating the images.

Despite that the design is optimized for microcirculatory imaging, as shown in Fig. 1(b), it is not easy to extract physiological features from SDF videos, such as the blood flow velocity, for several reasons: (1) Subsurface scattering: scattering of light on the path from the capillaries to the camera increases observed intensity of the vessels, reducing contrast of the images; (2) Defocus: capillaries are embedded at varied depths within the tissue while the depth of field of the camera is fixed to obtain desired magnification. So some capillaries in the field of view appear blurred, making their features more difficult to estimate; (3) Sensor noise that further reduces quality of images; (4) Limited texture: low diameter capillaries of interest comprise only a small part of the image, most of it is occupied by tissue without substantial texture and in addition, plasma in the capillaries is transparent, reducing texture in the frames even further, so traditional texture-based image feature extraction methods will likely fail; (5) Sensor drift during video capture: field of view changes due to the motions induced by heart beat and respiration of the subject and movement of the device itself, relative to the observed tissue.

In this paper, we present an end-to-end, automated framework for real-time analysis of micro-circulation including vessel detection, heart rate, breathing rate, blood flow velocity estimation as well as variations of flow distributions over time during bleeding and resuscitation stages. Our work can enable new research in critical care, helping 


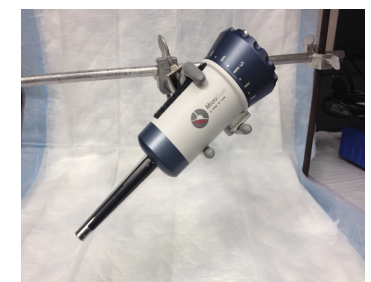

(a)

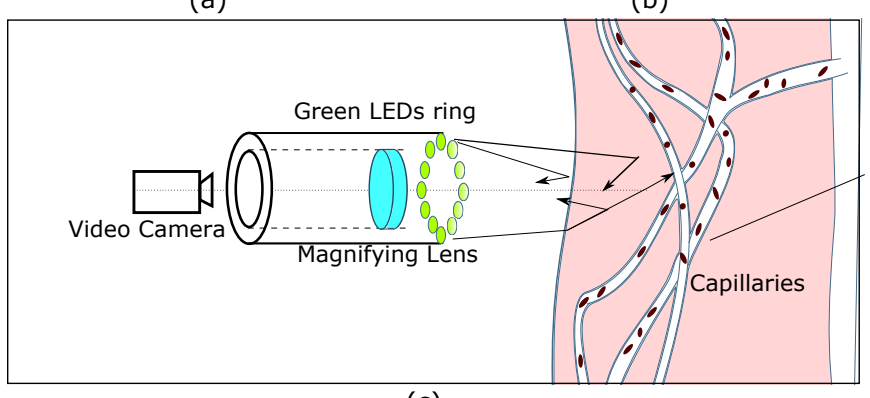

(c)

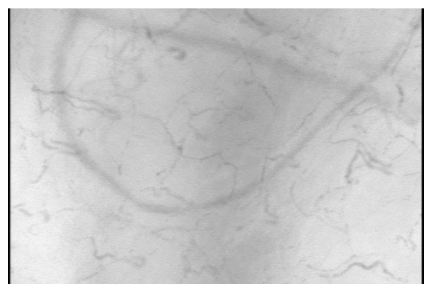

(b)
Figure 1: Sidestream Dark Field Imaging[6]. (a) Portable SDF imaging device used for microcirculatory monitoring. (b) One frame of the microcirculatory video. (c) The LEDs, arranged and optically isolated around the lens system, emit light optimized for red blood cell absorption. Due to defocus, subsurface scattering of light, sensor noise, sensor drift and limited texture of the tissue, it is hard to extract physiological features from the SDF video.

correlate heart rate and breathing cycle with flow distributions and studying effects of interventions and protocols in real-time for bed-side patient care. In comparison, most of the prior work either included significant manual interactions and were not real-time, or were tailored to high quality $2 \mathrm{D}$ images or $3 \mathrm{D}$ volumes that do not work for SDF videos.

The underlying principle of our approach is that diagnostically useful information must be extracted quickly, enabling the user to make determinations about microcircluatory flow in real time, rather than off line as is done currently, and ultimately enable making clinical decisions instantly at the bedside. To this end, we present a framework consisting of multiple stages including video stabilization, enhancement, micro-vessel extraction and automatic estimation of the micro blood flow statistics from SDF videos.

Our method has been used in a critical care experiment conducted carefully to analyze the microcirculatory blood flow of subjects in different health conditions. In the experiment, healthy pigs have been anesthetized and subjected to induced slow bleeding ( $20 \mathrm{ml} / \mathrm{min}$ ) for about 2 hours. Then the subjects were fluid resuscitated to expand the plasma volume. Microcirculatory videos were captured at different stages of the experiment to monitor changes in the micro blood flow. 96 videos of 18 pigs were collected using a SDF imaging device for each bleeding/resuscitation stage.
Our method was then applied to extract physiological information from the videos. As a result, the extracted informative microcirculatory features form distributions that are consistent with the intuition of expert clinicians.

\section{Related work}

Image based microcirculatory blood flow measurements have been studied using laser speckles [1, 2]. More recently, skin perfusion measurement based on laser speckle was proposed in [12]. Instead of images or videos of the microcirculatory blood flow, these methods leverage complex speckle patterns. In Sidestream Dark Field imaging system [6], microcirculatory blood flow is analyzed while the labeling of capillaries is typically done manually [4].

The vessels in the image are often detected as centreline structures $[14,17,13,9,11]$ either by using filters [17, 9], intensity profiles [14, 11], or trained regressors [13]. Then, level-set methods are used to locate the centreline more precisely [8, 18].

In [15], various optical flow approaches are studied. It was shown that by using an objective with a non-local term, the classical optical flow formulations can achieve competitive results. For motions of deformable objects, the motion estimation problem is often formulated as optimization solved by inverse compositional image alignment [10], supervised-learning of descent direction and data-driven descent [16]. In our case, with high level of noise, highly deformable blood flow patterns, and small dimensions of capillaries, it is very difficult to track the flow on a frameby-frame basis.

To get motions that are more obvious and easier to detect, video motion magnification method has been proposed in [20]. Extensions have been put forward to either reduce the noise in the motion magnified video [20] or achieve realtime running speed [19]. Because of the high level noise in the SDF videos, applying any of those methods directly would likely amplify the noise as well.

\section{Micro-vessel Extraction from Video}

The contrast of the SDF images is greatly reduced by the presence of the subsurface scattering and sensor noise. This makes it difficult to detect the capillaries from any single frame in the video. One option is to detect the capillaries from the minimal image, where the values of the pixels are set to the minimal intensity across frames at that pixel location. However, the input videos are not stable because of motions introduced by heartbeat, respiration, and sensor position drift. So we need to stabilize the video before extracting vessel skeletons. 


\subsection{Video Stabilization}

After motion due to heartbeat, breathing and sensor position drift is eliminated, the stabilized video will mainly consist of the blood flow in the capillaries. For efficiency considerations, we base stabilization on motions of the patches that are matched between frames using template matching. Since the microcirculatory videos are captured carefully to avoid unnecessary motion of sensor relative to subject, frame-to-frame changes are limited and smooth. Thus the correspondence between patches in different frames can be estimated. In addition, patch-based stabilization method enables including variations of the patch motions in a frame introduced by deformations of the tissue.

Because the videos are effectively textureless in most parts of the frames, we need to select the optimal patches for finding correspondence in the stabilization process. In our method, we select the patches in which the variance of intensities is above a pre-set threshold such that the selected patches include enough texture for matching. We use the locally affine models to deal with the non-rigid motion of the tissue introduced by heartbeat and breathing.

\subsection{Vessel skeleton extraction}

After stabilization, we have registered frames from which the skeletons of vessels can be extracted. However, as shown in the first column of Fig. 2, due to subsurface scattering and imaging noise, the contrast in individual frames is too low for extracting vessel segments. Even worse, the transparent plasma travelling through the capillaries may make vessels invisible in some segments of a frame. So we first need to produce a vessel-enhanced image. Based on the fact that the capillaries with red blood cells are usually darker in the frames, we can take the minimal value of each pixel across all frames to achieve that goal. This method works under assumption that for every pixel of the vessel there is at least one frame in which a red blood cell passes through it. This assumption is true for most cases since the duration of microcirculatory videos ( 20 seconds) is long enough for the red blood cells to pass through all the active vessels in the frame.

Then the vessel enhanced image is denoised by applying anisotropic diffusion filtering. It does not only reduce the imaging noise, while leaving the edges in the vesselenhanced image unharmed, but it also smoothes the parts of the image along the structures between the edges. This yields vessel segments with a smooth appearance so they can be detected more easily. The filtered vessel enhanced images are shown in the second column of Fig. 2.

To detect the vessel skeletons, we first estimate the Hessian matrix for each pixel in the vessel enhanced image. Then the profile for each pixel is extracted along the direction of the eigenvector of Hessian corresponding to the largest absolute eigenvalue. The pixel will be selected as a vessel skeleton pixel if the profile has a groove in the middle and increases towards both sides of the groove. To find the vessel skeletons with such profile, we use the method proposed in [14] that was designed to find the centreline of curvilinear structures.

Let $\mathbf{n}=\left(n_{x}, n_{y}\right)$ with unit length be the direction in the eigenvector of the Hessian Matrix $H$ corresponding the largest eigenvalue. The second-order Taylor expansion of pixel at $\mathbf{x}$ along $\mathbf{n}$ is given by:

$$
p(t)=r+r_{n} t+\frac{1}{2} r_{n n} t^{2}
$$

where $p(t)$ is the pixel intensity at the position $\mathbf{x}+t \mathbf{n} ; r, r_{n}$ and $r_{n n}$ are the pixel intensity at $\mathbf{x}$, the first-order derivative of the intensity in the direction $\mathbf{n}$ and the second-order derivative of the intensity in the direction $\mathbf{n}$ respectively. For a profile across the vessel, the center of the groove is located at the zero crossing of the first derivative of the profile:

$$
t=-\frac{r_{n}}{r_{n n}}=-\frac{\nabla \mathbf{r}^{T} \mathbf{n}}{\mathbf{n}^{T} H \mathbf{n}}
$$

where $\nabla \mathbf{r}$ is the gradient of the image at $\mathbf{x}$. In the image coordinates, the offset of the zero crossing from $\mathbf{x}$ is $\left(p_{x}, p_{y}\right)=\left(t n_{x}, t n_{y}\right)$, with $t$ estimated in Equation 2. The pixel $\mathbf{x}$ is on the vessel skeleton if $\left|p_{x}\right| \leq \frac{1}{2}$ and $\left|p_{y}\right| \leq \frac{1}{2}$. To eliminate the falsely detected vessels introduced by imaging noise, we use the maximum eigenvalue of the Hessian matrix to select the detected vessel skeletons.

The example results of the skeleton extraction are shown in Fig. 2. By comparing with the vessels manually labeled by human experts, we find that our vessel skeleton extraction method is able to locate most of these vessels. Although there is a potential for a few missing and a few false detections, the main objective of our work extraction of informative statistics of the physiological importance, and not the analysis of the individual vessels - should not suffer much. Hence, the obtained skeletons can be used as reliable inputs to the subsequent processing steps.

\subsection{Comparisons}

We have compared performance of our methods against other vessel extraction methods. For vessel skeleton extraction, our method yields a recall of $87.90 \%$ and false alarm rate of $0.65 \%$. In comparison, EF filters [5] on the minimal frame followed by adaptive thresholding of the filter response yields $51.86 \%$ and $0.36 \%$ in accuracy and false alarm rate. 2D OOF filters [9] on the minimal frame followed by adaptive thresholding of the filter response yields $70.79 \%$ and $1.29 \%$. Note that these alternative techniques fare worse when applied to the original videos without subsurface scattering reduction. 

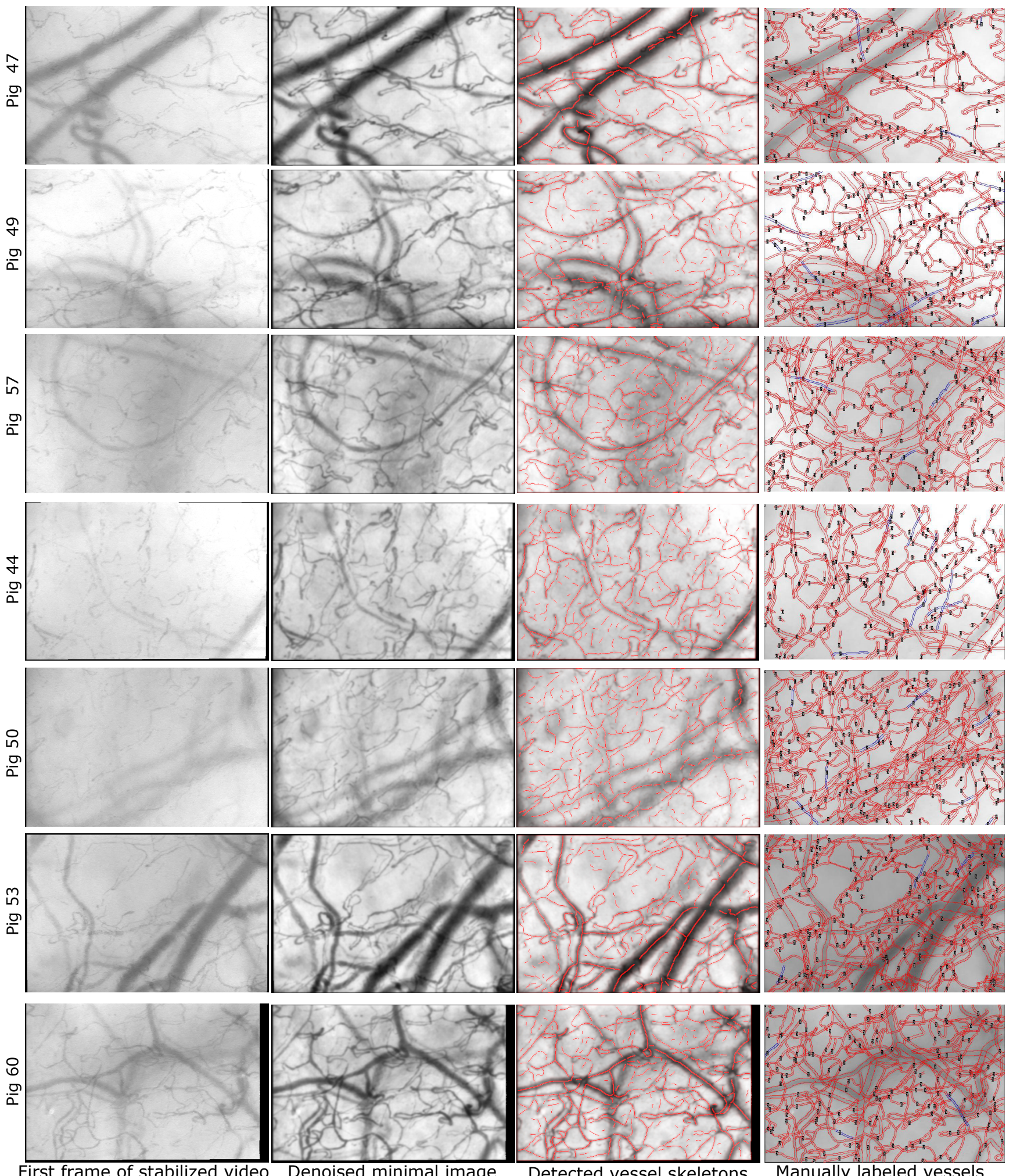

Detected vessel skeletons

Manually labeled vessels

Figure 2: The vessel skeletons are extracted from the minimal image across the frames. First column: the first frames of the videos. Due to subsurface scattering and transparency of plasma, it is hard to detect capillaries from a single frame. Second column: denoised minimal image across all of the $N$ frames (in our case $N=200$ ). The contrast is enhanced and the vessels are more detectable so that the vessel skeleton extraction methods can work. Third column: extracted vessel skeletons. Fourth column: manually painted vessels. The index and status of subjects in each row: Pig 44, before resuscitation; Pig 50, before resuscitation; Pig 53, end of baseline; Pig 60, end of baseline. 


\section{Physiological Measurements from Video}

Heartbeat and respiration rates can be obtained as side products of the video stabilization process. Those physiological measurements can be used, along with the microcirculatory blood flow parameters, to further aid diagnosis and patient monitoring. As the observed motion introduced by the heartbeat and breathing also depends on the location where the microcirculatory videos are taken, the measured motion can be used as a guidance for the clinician to determine the location of target tissue considered for diagnosis. In addition, although specialized monitoring of heart and respiratory rates already exists in clinical practice, it is not known whether and how their variations impact physiology of tissue blood flow. Estimation of these vital signs directly in the field of view provides an opportunity to accurately study these interactions in a live subject concurrently with flow information, and to further clinical knowledge.

We decompose the observed cross-frame motion into heartbeat and respiration motions based on their frequencies. More specifically, the respiratory effect is the motion component in the $[.1, .5] \mathrm{Hz}$ frequency range in the Fourier transform of the averaged observed motions of patches in the un-stabilized video; and the heartbeat is the motion component in the $[.5,5] \mathrm{Hz}$ frequency range. In these frequency ranges, the frequencies of the heartbeat and respiration motions are determined as the frequencies where the local maxima of magnitude in the Fourier domain occur. The magnitudes depend on the status of the subject and the location where the video is taken. For Pig 42, as shown in Fig. 3(a), most of the observed motion is due to the heartbeats. For Pig 44 at the end of bleeding, both the respiration and heartbeat motions are more significant. For Pig 44 before bleeding, the sensor drift dominates the observed motion, while the other two components can still be reliably identified. This last observation has important practical implication, since apparently the perfect stabilization of the sensor probe against the subject tissue is not necessary for extracting reliable physiological information from SDF imaging videos.

After the videos are stabilized, we estimate local blood flow velocity using skeletons to identify individual vessels. Even though we have now vessel-enhanced images with improved contrast, it is still difficult to determine blood flow from video, because signal to noise ratios are still low with the effects of subsurface scattering and high imaging noise. To make the blood flow more detectable, we use the motion magnification method proposed in [20]. In general, motion magnification is achieved by amplifying the frequency components within a given range for each voxel in the video. This method is based on the intuition that for one point in the video with repeating motion, the frequency of change in the intensity depends on the speed of motion that passes through that point. Motion is then amplified by magni-

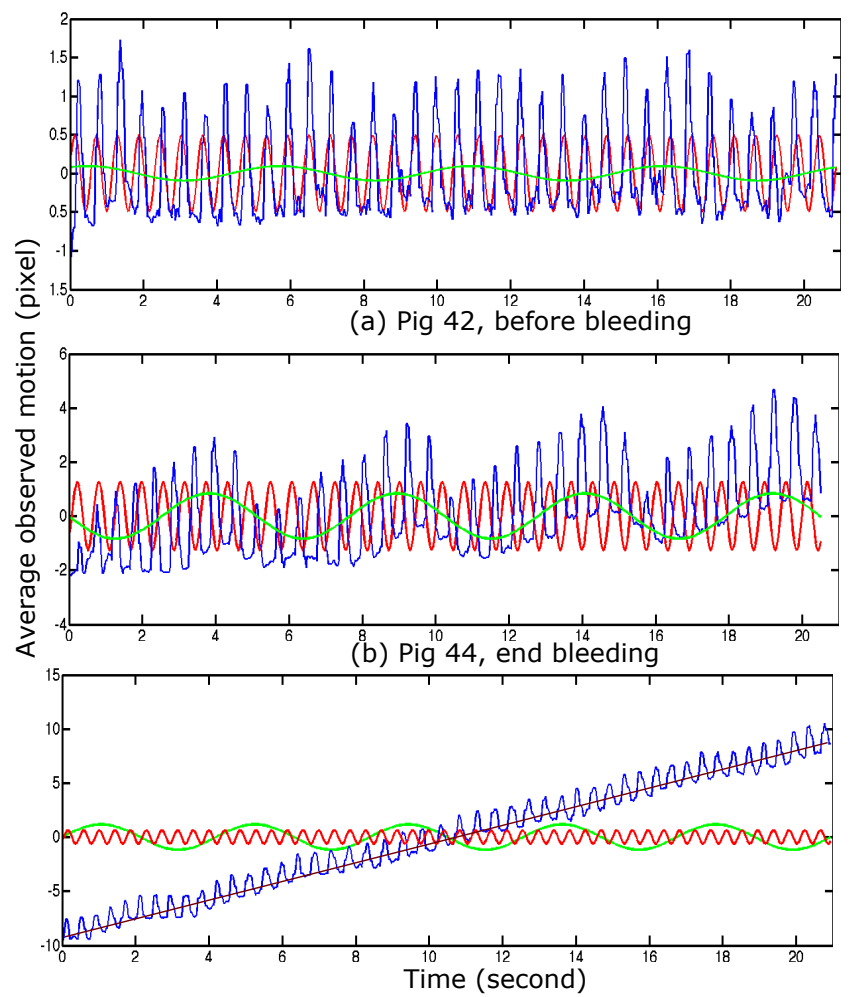

(c) Pig 44, before bleeding

Figure 3: We estimate the heartbeat rate and respiration rate by extracting the periodic heartbeat and respiration motions respectively from the averaged observed motion across the frame. (a) Pig 42 at the end of baseline (before bleeding). Most of the observed motion is due to the heart beat. (b) Pig 44, end of bleeding. Both heartbeat induced motion and breathing motion are obvious. (c) Pig 44, baseline. In (c), the sensor position drift (shown in brown) dominates the averaged observed motion, but the physiologic components can still be clearly identified.

fying the frequency component corresponding to it. The first frames in the original video and the motion magnified video are shown in Fig. 4. Note that the contrast between the plasma and red blood cells is enhanced in the motion magnified video. On the other hand, background noise has also been magnified since the frequency ranges of noise and blood flow overlap.

Blood flow velocity is estimated from the motion magnified video and the vessel skeletons. There is significant variation in flow velocity across vessels of different shapes and sizes, as well as due to physiological variations. This makes the optical flow method hard to work well in our case. One example is shown in Fig. 5. For a motion magnified video, the color coded optical flows estimated using the method in [15] for two consecutive frames are shown in Fig. 5(c) and Fig. 5(d). The corresponding vessel enhanced image for the 


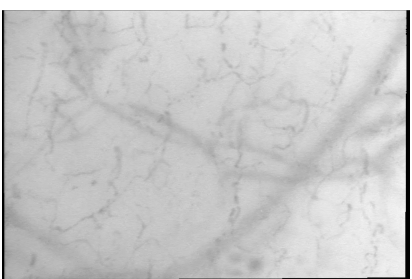

(a) Orginal video

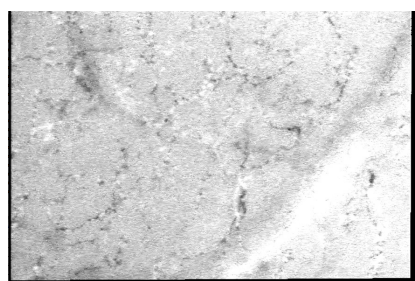

(b) Motion magnified video
Figure 4: The first frame in the original video and the motion-magnified video. Noise in the original video is magnified along with the blood flow motion.

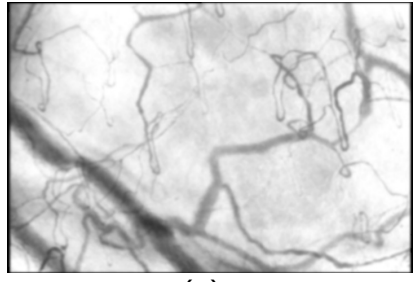

(a)

(c)

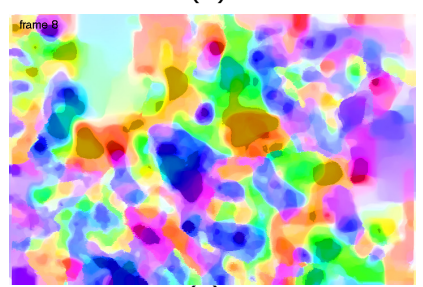

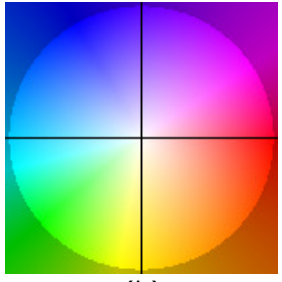

(b)

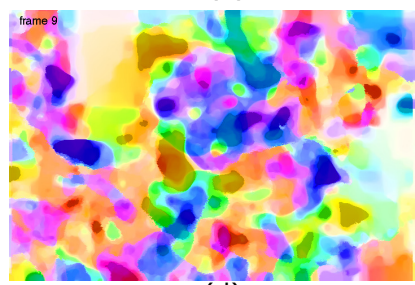

(d)
Figure 5: The blood flow velocity estimation using optical flow [15]. (a) The vessel enhanced image. (b) The color wheel showing colors corresponding to directions and magnitudes of optical flow. (c) The color coded optical flow for frame 8. (d) The color coded optical flow for frame 9.

video is shown in Fig. 5(a). Due to the imaging noise and difficulty in tracking the flow, the estimated optical flows for the two consecutive frames are very inconsistent, making the optical flow estimation unreliable.

Since the diameters of capillaries in the microcirculatory videos are small, the blood flow motion in the video can be reliably approximated by $1 \mathrm{D}$ motion along the vessel skeletons. Thus the blood flow velocity is estimated from the Epipolar-Plane Image (EPI) along the vessel skeleton length. As the blood flow velocity along a vessel is relatively constant, the flows are manifested as stripe patterns in the EPI image, with the slopes indicating the velocity of the flow, as shown in Fig. 6(b). Based on the property of the Fourier transform, the rotation of a pattern by an angle in the image domain will yield a rotation in the Fourier domain with the same angle. Thus we can estimate the velocity of the blood flow by finding the dominant orientation in the Fourier domain of the EPI image. This dominant orientation can be found by fitting the line passing through the

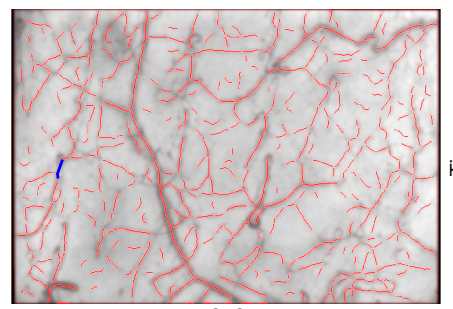

(a)

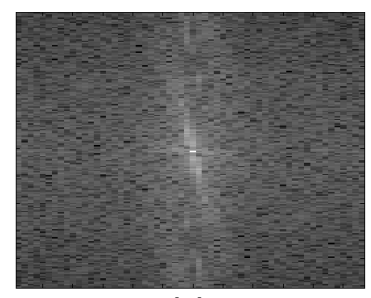

(c)

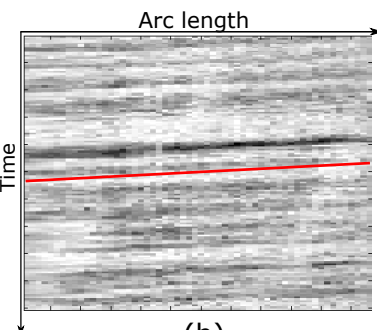

(b)

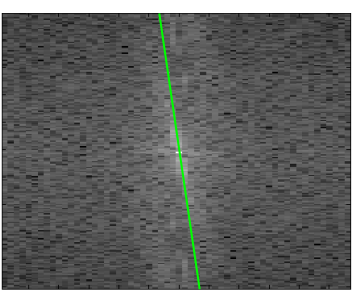

(d)
Figure 6: Blood flow velocity estimation. (a) The extracted vessel skeletons. The vessel segment for which the flow velocity is estimated is colored in blue. (b) The EPI image of the blue colored vessel segment in (a). (c) The Fourier Transform of the EPI image. (d) The dominant orientation of the Fourier Transform is plotted in green. The corresponding line showing the dominant orientation in the EPI image is plotted in red in (b).

origin, such that the second-moment inertia is minimized. This is a standard inertia minimization problem. Please refer to the supplementary material for more details.

The fitted line with the optimal angle indicating the dominant orientation in the Fourier domain is plotted in Fig. 6(d) in green. According to the rotation property of the Fourier Transform, the dominant orientation in the EPI image is the estimated orientation in the Fourier domain rotated clockwise by 90 degrees, as shown in Fig. 6(b). Although there are various orientations of patterns in the EPI image because of temporal and spatial fluctuations of blood flow velocity, the proposed method is still able to extract the dominant orientation, and therefore estimate the velocity.

In our experiments, the proposed method fits the task better than the optical flow approach because it takes into account data from multiple consecutive frames, while the optical flow method usually takes into account only two consecutive frames. Instead of measuring the blood flow velocity in one specific frame, our method actually measures the average blood flow velocity from multiple frames, which is more robust to the noise in the videos. By varying the extent of averaging, we can control temporal resolution of blood flow velocity estimation. It must be however noted that the frame-to-frame noise in our videos limits in practice the minimal time scales of this estimation. 


\section{Critical Care Case Studies}

In this section we will relate the estimated blood flow velocity distribution across all vessel segments detected in the field of view, to the status of the test subjects in the bleed and resuscitation phases of the experiments in order to evaluate consistency of our method with knowledge and intuition of expert clinicians.

The critical care experiment procedure is shown in Fig. 7. All experiments were performed in accordance with NIH guidelines under protocol approved by the Institutional Animal Care and Use Committee of the University of Pittsburgh. Yorkshire Durock pigs (average weight of $30.6 \mathrm{~kg}$ ) were fasted overnight prior to the study. Anesthesia and the surgical preparation have been performed following procedures described in [7].

Briefly, following induction of general anesthesia and endotracheal intubation, arterial and central venous catheters were inserted and the animals allowed to stabilize for 30 minutes. During this time the SDF probe attached to a vise clamp was positioned in the pigs mouth under the lounge to visualize the sublingual microcirculation. Care was taken to obtain a long-term stable image with minimal pressure artifact and good visualization of the microcirculation as defined by the optimal focal length and illumination to visualize the largest number of capillaries within the viewing frame as previously recommended in [3].

At the end of the baseline period the initial video was collected (Baseline). All videos were 20 seconds in length at 10 frames per second. Then the pigs were bled form the arterial catheter at a fixed rate of $20 \mathrm{ml} / \mathrm{min}$ until the mean arterial pressure decreased to $30 \mathrm{mmHg}$. Once at this pressure, bleeding was stopped and a second video was captured (EndBleed). The subject was kept in this hypotensive state for 90 minutes with video images captured at 60 minutes into the hypotensive state (AfterBleed) and again at 90 minutes (BeforeResusc). Then the pigs were fluid resuscitated with Hextend (equal volume to shed blood) at 60 $\mathrm{ml} / \mathrm{min}$. At the end of this fluid resuscitation period another video was captured (EndHextend). Then the animal was further resuscitated in a protocolized fashion as previously described with more fluid if the cardiac output was less than baseline and norepinephrine if mean arterial pressure was less than baseline for an additional 120 minutes and a final video image was taken (AfterHextend). Since many animals became unstable before 90 minutes of hypotension or did not survive 120 minutes after the start of resuscitation, some animals did not have BeforeResusc and AfterHextend time point videos collected.

From the point of view of current knowledge of physiology of the observed processes, as the blood pressure decreases due to bleeding, a general reduction in blood flow velocity is expected. It should manifest by a shift of the distribution of velocities across vessels towards lower values

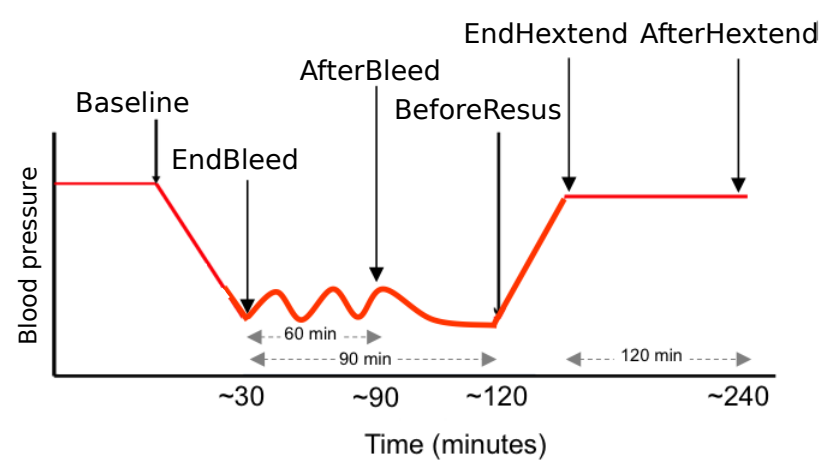

Figure 7: Setup of the experimental procedure. In the experiment, 18 healthy pigs have been anesthetized and subjected to bleed for 2 hours. Then the subjects are fluid resuscitated to expand the plasma volume. A 20 seconds microcirculatory video is captured at each stage of the experiment to monitor changes in the micro blood flow.

of velocity. Although resuscitation should intuitively led to an increase of microcirculatory flow, the temporal relation between restoration of arterial pressure and cardiac output to microcirculatory flow is complex and not yet fully understood. Still, one would expect that if resuscitation efforts were successful, that microcirculatory blood flow would return to baseline values.

The distributions of the blood flow velocity estimated from the corresponding videos are consistent with the above intuition. The results for five pigs are shown in Fig. 8. For better visualization, in the right column we show only the results for three most important stages in the process. For Pig 44 and Pig 60, the blood flow in the capillaries diminishes after bleeding as the blood pressure and the vitality of the pig deteriorate. This change has been reflected in the figure as the flow velocity distribution, shown in the green curve, squeezes towards lower values. In addition, the number of capillaries with slow flow velocity decreased after resuscitation as compared to the after bleed phase. This is consistent with physiologic expectations, and represents the opening of capillary beds that were previously closed probably due to insufficient input pressure during shock. Given that this protocol was intended to study the individual responses of each animal to hemorrhage, blood volume shed was different between animals. Pig 44 and 60 had $534 \mathrm{ml}$ and $760 \mathrm{ml}$, respectively, which represented $23 \%$ and $36.7 \%$ of their calculated total blood volume, respectively. This analysis also demonstrated how for example pig 44 had a lower relative increase in capillaries with slow flow, than pig 60, which is consistent with having had a less intense response, to a less intense injury.

For Pig 47, the difference in the blood flow velocity distributions between the baseline and after bleed is smaller 
than those for Pig 44 and Pig 60. This is because more probe pressure on the tissue was introduced during capturing the microcirculatory video for Pig 47 in the baseline stage, making the blood flow suppressed at that stage. Our method has reflected such measuring artifacts during capture. For Pig 55, there are only 5 stages in total since the pig died before last stage.

\section{Conclusion}

We presented a multi-stage framework for processing microcirculatory videos automatically and in real time. The processing stages include video stabilization, image enhancement, and micro-vessel extraction, in order to automatically estimate statistics of the micro blood flow captured in SDF videos. We applied our method to analyze changes in microcirculation in test animals at different stages of induced bleeding experiment, including before, during and after bleeding as well as after resuscitation. The results show that by using image augmentation and continuous video sampling techniques, reliable microcirculatory imaging processing can be automated and accomplished in real time despite the inherent challenges to microcirculatory flow quantization. The parameters described in this analysis represent novel metrics that should substantially improve the utility of SDF imaging to assess microcirculatory changes with disease and its treatment. In the future, we are going to improve the SDF imaging device such that more reliable and noisy-free data can be obtained. In addition, local features such as local flow velocity variation and intermittency of the flow will be studied to further enhance the functionality and clinical relevance of the framework.

\section{Acknowledgements}

This research was supported in parts by NSF awards 0964562 and 1320347, NIH grants 1K12HL109068-02 and 1R01GM082830-01A2, a Veterans Affairs Merit Award 1I01BX000566 and a Department of Defense grant DM102439.
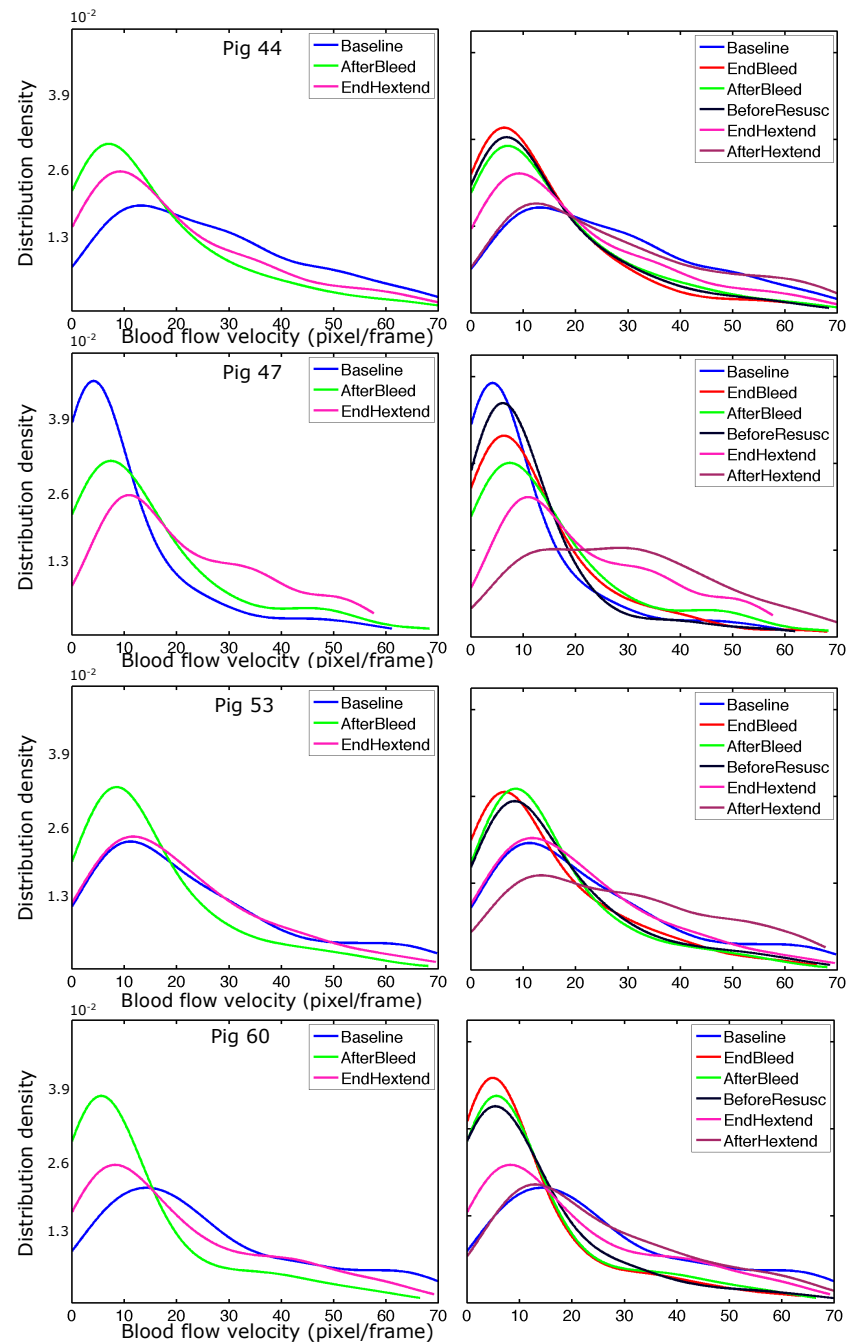

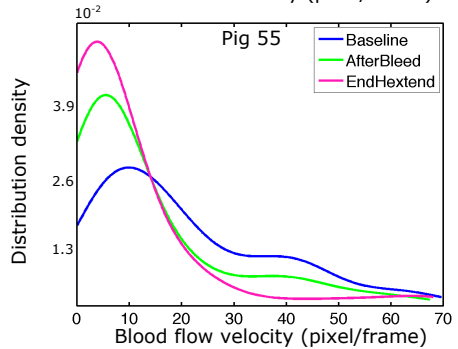

(a) Blood flow velocity distribution at three stages

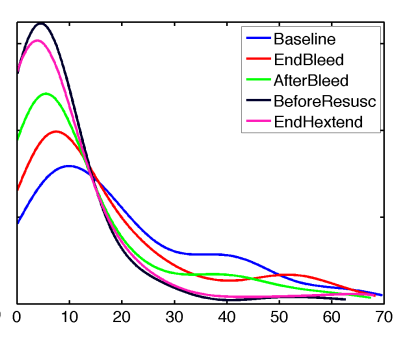

(b) Blood flow velocity distribution at all six stages
Figure 8: Estimated blood flow velocity distributions for pigs at different stages. For each plot, the x-axis is the blood flow velocity, in pixels per frame; the y-axis is the distribution density of vessels with corresponding flow velocities. (a) The blood flow velocity distributions at three key stages: baseline, end of bleed and right after resuscitation. (b) The blood flow velocity distributions for all six stages. The annotations for stages: Baseline (blue) - right before the bleeding procedure; EndBleed (red) - end of bleed; AfterBleed (green) - 60 minutes after EndBleed; BeforeResusc (black) - before resuscitation, 90 minutes after EndBleed, before the resuscitation procedure; EndHextend (purple) - end of resuscitation procedure; AfterHextend (yellow) - 120 minutes after EndHextend. The blood flow velocity decreases after the start of bleeding. 


\section{References}

[1] J. D. Briers and S. Webster. Laser speckle contrast analysis (LASCA): a nonscanning, full-field technique for monitoring capillary blood flow. . J. Biomed. Opt, Apr. 1996. 2

[2] H. Cheng, Q. Luo, Q. Liu, Q. Lu, H. Gong, and S. Zeng. Laser speckle imaging of blood flow in microcirculation. . Phys. Med. Bio, 2004. 2

[3] D. De Backer, S. Hollenberg, C. Boerma, P. Goedhart, G. Büchele, G. Ospina-Tascon, I. Dobbe, and C. Ince. How to evaluate the microcirculation: report of a round table conference. Critical Care, 11(5):R101, 2007. 7

[4] J. G. Dobbe, G. J. Streekstra, B. Atasever, R. van Zijderveld, and C. Ince. Measurement of functional microcirculatory geometry and velocity distributions using automated image analysis. Medical \& biological engineering \& computing, 46(7):659-670, Apr. 2008. 2

[5] A. F. Frangi, W. J. Niessen, K. L. Vincken, and M. A. Viergever. Multiscale vessel enhancement filtering. MICCAI, pages 130-137, 1998. 3

[6] P. T. Goedhart, K. M, R. Bezemer, J. Merza, and C. Ince. Sidestream Dark Field (SDF) imaging:a novel stroboscopic LED ring-based imaging modality for clinical assessment of the microcirculation. In Optics Express, pages 1-14, Dec. 2007. 1,2

[7] H. Gómez, J. Mesquida, L. Hermus, P. Polanco, H. Kim, S. Zenker, A. Torres, R. Namas, Y. Vodovotz, G. Clermont, J. Puyana, and M. Pinsky. Physiologic responses to severe hemorrhagic shock and the genesis of cardiovascular collapse: can irreversibility be anticipated? . Journal of Surgical Research, Nov. 2012. 1, 7

[8] M. S. Hassouna and A. A. Farag. MultiStencils Fast Marching Methods: A Highly Accurate Solution to the Eikonal Equation on Cartesian Domains. IEEE Transactions on Pattern Analysis and Machine Intelligence, 29(9):1563-1574, 2007. 2

[9] M. W. Law and A. C. Chung. Three dimensional curvilinear structure detection using optimally oriented flux. Proceedings of European Conference on Computer Vision (ECCV), pages $368-382$, 2008. 2, 3

[10] I. Matthews and S. Baker. Active appearance models revisited. International Journal of Computer Vision, 60(2):135164, Nov. 2004. 2

[11] A. M. Mendonça and A. Campilho. Segmentation of retinal blood vessels by combining the detection of centerlines and morphological reconstruction. Medical Imaging, IEEE Transactions on, 25(9):1200-1213, 2006. 2

[12] G. Satat, C. Barsi, and R. Raskar. Skin perfusion photography. In Proceedings of IEEE International Conference on Computational Photography (ICCP), pages 1-8, 2014. 2

[13] A. Sironi, V. Lepetit, and P. Fua. Multiscale Centerline Detection by Learning a Scale-Space Distance Transform. In Proceedings of IEEE Conference on Computer Vision and Pattern Recognition (CVPR), pages 2697-2704, 2014. 2

[14] C. Steger. An Unbiased Detector of Curvilinear Structures. IEEE Transactions on Pattern Analysis and Machine Intelligence, pages 1-13, Feb. 1998. 2, 3
[15] D. Sun, S. Roth, and M. J. Black. Secrets of Optical Flow Estimation and Their Principles. Proceedings of IEEE Conference on Computer Vision and Pattern Recognition (CVPR), pages 1-8, Mar. 2010. 2, 5, 6

[16] Y. Tian and S. G. Narasimhan. Globally Optimal Estimation of Nonrigid Image Distortion. International Journal of Computer Vision, 98(3):279-302, July 2012. 2

[17] E. Turetken, C. Becker, P. Glowacki, F. Benmansour, and P. Fua. Detecting Irregular Curvilinear Structures in Gray Scale and Color Imagery Using Multi-directional Oriented Flux. In Proceedings of IEEE International Conference on Computer Vision (ICCV), pages 1553-1560. IEEE, Nov. 2013. 2

[18] R. Van Uitert and I. Bitter. Subvoxel precise skeletons of volumetric data based on fast marching methods. Medical Physics, 34(2):627, 2007. 2

[19] N. Wadhwa, M. Rubinstein, F. Durand, and W. T. Freeman. Riesz pyramids for fast phase-based video magnification. In Proceedings of IEEE International Conference on Computational Photography (ICCP), pages 1-10, 2014. 2

[20] H.-Y. Wu, M. Rubinstein, E. Shih, J. Guttag, F. Durand, and W. Freeman. Eulerian video magnification for revealing subtle changes in the world. ACM Transactions on Graphics (TOG), 31(4):1-8, July 2012. 2, 5 